\title{
Chaotic dynamics in spinning shafts with non-constant rotating speed described by variant Lyapunov exponents
}

\author{
Fotios Georgiades [0000-0002-2784-6976] \\ School of Engineering, College of Science, University of Lincoln, Lincoln, UK \\ fgeorgiadisalincoln.ac.uk
}

\begin{abstract}
The dynamics of spinning shafts with non-constant rotating speed is described by a nonlinear system that under certain conditions might exhibit also chaotic behavior. In this article chaotic dynamics of the spinning shaft is examined. Initially, the trajectories in phase space around the equilibrium manifolds are determined. Then by choosing a set of initial conditions, nearby to an equilibrium, corresponding to eigenvalues of the Jacobian with a nonzero real part, identification of chaos is examined. Approximations of the trajectory, with the linearization curves around the equilibria, are defined and they are good in a region very close to the associated equilibrium point. It is shown that the eigenvalues, as Lyapunov exponents indicators, are not parameter dependent but state dependent. The eigenvalues of the linearized system within an orbit are varying from positive to zero, therefore the Lyapunov exponent is not defined through this limit as an explicit number but variant. The existence of eigenvalues with positive real parts in certain parts of the orbit is an indication of chaos since it shows a divergence of nearby orbits. One orbit starting from an initial condition which corresponds to eigenvalues with positive real part is crossing the threshold and pass to points that the eigenvalues with zero real parts, therefore this 'threshold' is not discriminating chaotic with regular regions as expected. The variant positive Lyapunov exponents have been examined also with numerical investigations and it is an indication of chaos. The Poincare section indicates irregular motion and the approximated Information Entropy is relatively high, and both are indicating chaos. It should be highlighted that this is a mechanical system with variant real parts of eigenvalues as Lyapunov exponents within one orbit and the threshold is insufficient to distinguish chaotic from regular regions. Further work is needed to determine the chaotic regions of the spinning shaft. Further developments in the mathematics of nonlinear dynamical systems associated with the equilibrium manifolds are needed to examine the significance of variant Lyapunov exponents for this kind of systems. Also, the necessity to reexamine the validity of existing algorithms and the development of new ones for the determination of variant Lyapunov exponents, become evident.
\end{abstract}

Keywords: Variant Lyapunov exponent, Chaos, Spinning shaft. 


\section{Introduction}

There is a significant number of articles focused in dynamics of rotating structures with constant rotating speed but the examination of dynamics of rotating structures with a non-constant rotating speed which occurs during spin-up/down operation is very limited [1-5]. In Ref. [4], the equations of motion of a spinning shaft as Euler-Bernoulli isotropic beam has been derived and dynamic analysis is performed, noted that the model has a similar form with this one obtained in Ref. [3] about rotating blades. In Ref. [5] the Equilibrium Manifolds (EMs) have been determined with their associated normal modes and stability is examined. Also, there is a vast number of articles examining chaotic dynamics near specific equilibrium points but articles relevant to equilibrium manifolds with the examination of points that the linearized solution is changing nature with state space dependent eigenvalues are very limited and few relevant comments can be found in [6].

This article is a continuation of the work done in Ref. [5], focused in chaotic motions in the nonlinear dynamics of the spinning shaft, using typical technique applied in nonlinear dynamics which is the linearization around EM. Firstly, the trajectories arising from linearization around some EMs are determined, and secondly using direct numerical integration the validity of the solutions with the associated eigenvalues is examined. Also using numerical methods, the notion of variant Lyapunov exponent and the chaotic behaviour in the dynamics of the spinning shaft are examined by means of the determination of Poincare section, the Lyapunov Exponents $(L E)$ and the approximated Information Entropy for a specific set of Initial Conditions (ICs).

\section{Trajectories around equilibrium manifolds}

A spinning shaft (with length- $L$, internal- $D_{i}$ and external diameter $D_{o}$ ) with a non-constant rotating speed made of isotropic material (with density- $\rho$, Young's and Shear modulus being $E, G$ respectively) modelled as Euler-Bernoulli beam is considered. The equations of motion describing the dynamics are given in Ref. [4] and in Ref. [5] by neglecting the equation of rigid body angular position then a restricted system is arising. The restricted system is not corresponding to specific energy function, therefore even if a linear counterpart of the restricted system exists, the examination of chaotic orbits through Melnikov theory would be rather complicated [7-9]. So, on this article as the first investigation of chaotic orbits on the restricted system, the linearization through perturbations around EM and examination of the associated real parts of the eigenvalues as indicators of $L E$ is used.

Herein the analysis will be restricted around the following EM,

$\mathrm{y}_{0}^{(1)}=\left(y_{0,1}, y_{0,2}, y_{0,3}, y_{0,4}, y_{0,5}, y_{0,6}, y_{0,7}\right)=\left(0,0,0, \dot{\theta}_{0}, 0,0,0\right)$ with $\dot{\theta}_{0} \in \mathbb{R}$,

and,

$\mathrm{y}_{0}^{(3)}=\left(y_{0,1}, y_{0,2}, y_{0,3}, y_{0,4}, y_{0,5}, y_{0,6}, y_{0,7}\right)=\left(0,0, q_{0, \phi}, \pm \omega_{T}, 0,0,0\right)$ with $q_{0, \phi} \in \mathbb{R},(2)$ 
namely $1^{\text {st }}$ and $3^{\text {rd }} \mathrm{EM}$ as mentioned in Ref. [5], whereas $y_{1}, y_{2}, y_{3}$, are the modal displacements in lateral bending motions and torsion respectively, $y_{4}$ is the rigid body angular velocity and the rest are the associated velocities of the modal displacements in the same order. Considering $\zeta_{i}\left(\zeta_{i}\right)$-perturbations for each generalized $i$-coordinate on the equilibriums for the $1^{\text {st }} \mathrm{EM}\left(3^{\text {rd }} \mathrm{EM}\right)$ and then after linearization, the system will be examined in the region of phase space that is defined by rigid body angular velocity of $\dot{\theta}_{0} \in\left(\omega_{b} \sqrt{(1-M)}, \frac{\omega_{b}(1-M)}{\sqrt{-M}}\right)$. The constants are given by,

$$
\begin{array}{lll}
m=\pi \rho\left(\frac{D_{o}{ }^{2}-D_{i}{ }^{2}}{4}\right), & I_{1}=\rho I=\rho \pi\left(\frac{D_{o}{ }^{4}-D_{i}{ }^{4}}{64}\right), & \omega_{b}=\sqrt{\frac{\pi^{4} \mathrm{EI}}{L^{2} \pi^{2} I_{1}+L^{4} \mathrm{~m}}} \\
\omega_{T}=\frac{\pi}{2 L} \sqrt{\frac{G}{\rho},} & F=\frac{2}{\pi} \sqrt{2 I_{1} L}, & M=-\frac{I_{1} \pi^{2}}{m L^{2}} .
\end{array}
$$

The linearization around the $1^{\text {st }} \mathrm{EM}\left(\mathrm{y}_{0}^{(1)}\right)$, leads to two fully decoupled systems of differential equations; one describing the two lateral bending motions and the other one describing the coupled torsional with rigid body motions which will be considered herein [5]. The linearization around the $3^{\text {rd }} \mathrm{EM}\left(\mathrm{y}_{0}^{(3)}\right)$ [5], lead to three systems of differential equations; one describing the two lateral bending motions fully decoupled from the equations that are describing the torsional with rigid body motions which will be considered herein [5].

The eigenvalues of the linearized system around the $1^{\text {st }}$ EM are given by [5],

$$
\left\{\lambda_{j}\right\}=\left[-\sqrt{\frac{I_{1} L\left(\dot{\theta}_{0}^{2}-\omega_{T}^{2}\right)}{\left(I_{1} L-F^{2}\right)}}, 0, \sqrt{\frac{I_{1} L\left(\dot{\theta}_{0}^{2}-\omega_{T}^{2}\right)}{\left(I_{1} L-F^{2}\right)}}\right]^{T}, j=1,2,3,
$$

which are real in case of $\dot{\theta}_{0}>\omega_{T}$. Therefore, based on the existing theory, there is a 'threshold' of $\dot{\theta}_{0}=\omega_{T}$, whereas for higher angular velocities the $L E$ from zero becomes positive [9]. The existence of the chaotic region for $\dot{\theta}_{0}>\omega_{T}$ and the transition to chaos around the region $\dot{\theta}_{0}=\omega_{T}$, will be examined. For $\dot{\theta}_{0}=\omega_{T}$, the $1^{\text {st }} \mathrm{EM}$ is approaching the $3^{\text {rd }} \mathrm{EM}$ which justifies that the examination must be performed considering both EM. The eigenvalues of the linearized system around $3^{\text {rd }}$ EM are purely imaginary or zero and they are given by [5],

$\left\{\mu_{j}\right\}=\left[-i \frac{2 \omega_{T} y_{0,3}}{\sqrt{\left(I_{1} L-F^{2}+y_{0,3}^{2}\right)}}, 0, i \frac{2 \omega_{T} y_{0,3}}{\sqrt{\left(I_{1} L-F^{2}+y_{0,3}^{2}\right)}}\right]^{T}, j=1,2,3$

The systems of differential equations which describe the motions around the $1^{\text {st }}$ and $3^{\text {rd }}$ EM will be used for the projection to the phase space by parametrizing time with respect to the perturbation in torsional position ( $\xi_{3}$ and $\zeta_{3}$ respectively) [5].

Using the linearized equations arising with $\xi_{i}$-perturbations around the $1^{\text {st }} \mathrm{EM}$ lead to [5],

$\frac{d \xi_{4}}{d \xi_{3}}=\frac{F\left(\dot{\theta}_{0}^{2}-\omega_{T}^{2}\right) \xi_{3}}{\left(I_{1} L-F^{2}\right) \xi_{7}}$ 
$\frac{d \xi_{7}}{d \xi_{3}}=\frac{I_{1} L\left(\dot{\theta}_{0}^{2}-\omega_{T}^{2}\right) \xi_{3}}{\left(I_{1} L-F^{2}\right) \xi_{7}} \Leftrightarrow \xi_{7}^{2}-\frac{I_{1} L\left(\dot{\theta}_{0}^{2}-\omega_{T}^{2}\right)}{\left(I_{1} L-F^{2}\right)} \xi_{3}^{2}=A$,

neglecting $\xi_{7} \neq 0$ which corresponds to the local extrema of perturbation of torsional position $\left(\xi_{3}\right)$, then in phase space the curves are given by,

$\xi_{7}^{2}-\frac{I_{1} L\left(\dot{\theta}_{0}^{2}-\omega_{T}^{2}\right)}{\left(I_{1} L-F^{2}\right)} \xi_{3}^{2}=A \Leftrightarrow y_{7}^{2}-\frac{I_{1} L\left(\dot{\theta}_{0}^{2}-\omega_{T}^{2}\right)}{\left(I_{1} L-F^{2}\right)} y_{3}^{2}=A$,

whereas the case of $\dot{\theta}_{0}>\omega_{T}$ corresponds to a family of hyperbolas with center $(0,0)$ in phase space, the case of $\dot{\theta}_{0}=\omega_{T}$ lead to a constant perturbation in torsional velocity $\left(\xi_{7}\right)$ and the last case of $\dot{\theta}_{0}<\omega_{T}$ corresponds to a family of ellipses with center $(0,0)$ in phase space. Using equation (9), the constant of integration A can be determined. Rearrangement of equation (9) lead to,

$\xi_{7}= \pm \sqrt{\frac{I_{1} L\left(\dot{\theta}_{0}^{2}-\omega_{T}^{2}\right)}{\left(I_{1} L-F^{2}\right)} \xi_{3}^{2}+A} \Leftrightarrow y_{7}= \pm \sqrt{\frac{I_{1} L\left(\dot{\theta}_{0}^{2}-\omega_{T}^{2}\right)}{\left(I_{1} L-F^{2}\right)} y_{3}^{2}+A}$,

then using (eq. 10) in (7) and after integration considering original system state space variables lead to,

$y_{4}=\dot{\theta}_{0} \pm \frac{F}{I_{1} L} \sqrt{\frac{I_{L} L\left(\dot{\theta}_{0}^{2}-\omega_{T}^{2}\right)}{\left(I_{1} L-F^{2}\right)} y_{3}^{2}+A}+B$,

which corresponds to the following families of orbits,

$\frac{\left(I_{1} L\right)^{2}\left(y_{4}-\dot{\theta}_{0}-B\right)^{2}}{F^{2} A}-\frac{I_{1} L\left(\dot{\theta}_{0}^{2}-\omega_{T}^{2}\right)}{A\left(I_{1} L-F^{2}\right)} y_{3}^{2}=1$,

whereas for $\dot{\theta}_{0}>\omega_{T}$ corresponds to a family of hyperbolas with centre $\left(\dot{\theta}_{0}+B, 0\right)$, for $\dot{\theta}_{0}=\omega_{T}$ lead to a constant rigid body angular velocity $\left(y_{4}\right)$ and finally for $\dot{\theta}_{0}<\omega_{T}$ lead to a family of ellipses with center $\left(\dot{\theta}_{0}+B, 0\right)$ in phase space.

In Ref. [5] the linearized namely $3^{\text {rd }}$ EM which corresponds to $\dot{\theta}_{0}=\omega_{T}$ is surrounded by periodic orbits, therefore the constant perturbations in torsional and rigid body angular velocities obtained from equations (10) and (11) respectively are not describing the orbits in this region. In this region of the orbit, a better approximation of the orbits can be obtained from the linearization of the namely $3^{\text {rd }} \mathrm{EM}$. The orbits associated with the $3^{\text {rd }} \mathrm{EM}$ around $\zeta_{i}$ - perturbations, by parametrizing time with the perturbation in torsional position $\left(\zeta_{3}\right)$ using equations obtained in Ref. [5], are determined. Firstly, the differential equation,

$\frac{d \zeta_{4}}{d \zeta_{7}}=\frac{F \zeta_{4}-\zeta_{7}}{\left(I_{1} L+y_{0,3}^{2}\right) \zeta_{4}-F \zeta_{7}}$,

neglecting the points of $\left(I_{1} L+y_{0,3}^{2}\right) \zeta_{4} \neq F \zeta_{7}$ which corresponds to the local extrema of perturbation of torsional velocity $\left(\zeta_{7}\right)$, is solved to obtain,

$\zeta_{4}=\frac{F \zeta_{7}}{\left(I_{1} L+y_{0,3}^{2}\right)} \pm \frac{\sqrt{-\left(I_{1} L-F^{2}+y_{0,3}^{2}\right) \zeta_{7}^{2}+C}}{\left(I_{1} L+y_{0,3}^{2}\right)}$. 
The constant of integration $\mathrm{C}$ can be determined using any pair of values in the following rearranged equation,

$\left(I_{1} L+y_{0,3}^{2}\right)^{2} \zeta_{4}^{2}+\left(I_{1} L+y_{0,3}^{2}\right) \zeta_{7}^{2}-2\left(I_{1} L+y_{0,3}^{2}\right) F \zeta_{4} \zeta_{7}-C=0$,

which is a $2^{\text {nd }}$ degree equation and it is forming an ellipse [10].

The other equation (considering $\zeta_{7} \neq 0$ ) is given by,

$\frac{d \zeta_{7}}{d \zeta_{3}}=2 \omega_{T} y_{0,3}\left(\frac{\left(I_{1} L+y_{0,3}^{2}\right)}{\left(I_{1} L-F^{2}+y_{0,3}^{2}\right)} \frac{\zeta_{4}}{\zeta_{7}}-\frac{F}{\left(I_{1} L-F^{2}+y_{0,3}^{2}\right)}\right)$,

and after using (eq. 14) and direct integration lead to,

$\zeta_{3}=\mp \frac{1}{2 \omega_{T} y_{0,3}} \sqrt{-\left(I_{1} L-F^{2}+y_{0,3}^{2}\right) \zeta_{7}^{2}+C}+D$,

or using state space variables of the original system and after rearrangement lead to the following family of curves in phase space,

$\left[2 \omega_{T} y_{0,3}\left(y_{3}-y_{0,3}-D\right)\right]^{2}+\left(I_{1} L-F^{2}+y_{0,3}^{2}\right) y_{7}^{2}=C$,

which is an ellipsis centered at $\left(y_{3}, y_{7}\right)=\left(y_{0,3}+D, 0\right)[10]$.

Solving equation (18) for perturbation of modal torsional velocity with respect to perturbation of modal torsional position lead to,

$\zeta_{7}= \pm \sqrt{\frac{C-\left[2 \omega_{T} y_{0,3}\left(\zeta_{3}-D\right)\right]^{2}}{\left(I_{1} L-F^{2}+y_{0,3}^{2}\right)}} \Leftrightarrow y_{7}= \pm \sqrt{\frac{C-\left[2 \omega_{T} y_{0,3}\left(y_{3}-y_{0,3}-D\right)\right]^{2}}{\left(I_{1} L-F^{2}+y_{0,3}^{2}\right)}}$,

and replacing (eq. 19) in (eq. 14) considering state space variables of the original system lead to,

$y_{4}=\omega_{T} \pm \frac{F}{\left(I_{1} L+y_{0,3}^{2}\right)} \sqrt{\frac{C-\left[2 \omega_{T} y_{0,3}\left(y_{3}-y_{0,3}-D\right)\right]^{2}}{\left(I_{1} L-F^{2}+y_{0,3}^{2}\right)}} \pm \frac{2 \omega_{T} y_{0,3}\left(y_{3}-y_{0,3}-D\right)}{\left(I_{1} L+y_{0,3}^{2}\right)}$,

and if it is written as a $2^{\text {nd }}$ order equation becomes evident that forms a family of ellipsis with center at $\left(y_{3}, y_{4}\right)=\left(y_{0,3}+D, \omega_{T}\right)[10]$.

\section{$3 \quad$ Numerical results-Discussion}

A 1-meter length shaft with internal and external radii $r_{i}=0.028 \mathrm{~m}$, and $r_{o}=0.03 \mathrm{~m}$, respectively, is considered. It is made of Stainless steel with density $\rho=7850 \mathrm{Kg} / \mathrm{m}^{3}$, Young modulus $E=200 \mathrm{GPa}$, shear modulus $G=76.9 \mathrm{GPa}$ and, Poisson's ratio $v=0.3$.

The ICs are $\left(y_{1}, y_{2}, y_{3}, y_{4}, y_{5}, y_{6}, y_{7}\right)=(0,0,0,4996.41,0,0,0.1)$ and correspond to a perturbation of the $1^{\text {st }} \mathrm{EM}$ for $y_{0,4}=\dot{\theta}_{0}=\omega_{T}+80 \mathrm{rad} / \mathrm{sec}$ whereas the trajectory around this point can be approximated by hyperbolas given by (eq. 9, 12). The total restricted system has been numerically integrated with time step (1.5e-5) for 
approximated 3997 orbits (approximating average orbital period $0.01251 \mathrm{sec}$ ). The full manifold superimposed with the plot of the EMs are depicted in Figure 1a, noted that the lateral bending motions are zero and in (fig. 1a) the total manifold is depicted. In Figure 1a the four points that the trajectory is approaching the $3^{\text {rd }} \mathrm{EM}\left(\dot{\theta}_{0}=\omega_{T}=\right.$ $4916.41 \mathrm{rad} / \mathrm{sec}$ ) are also indicated. The points are defined by the following sets of values; $\quad\left(y_{3}, y_{4}, y_{7}\right)=(0.001374,4916.411021,-2.686653)$ for the $1^{\text {st }}$ point, $\left(y_{3}, y_{4}, y_{7}\right)=(0.006003,4916.411603,2.686645)$ for the $2^{\text {nd }}$ point, $\left(y_{3}, y_{4}, y_{7}\right)=(-$ $0.001374,4916.411674,-2.686633)$ for the $3^{\text {rd }}$ point, and $\left(y_{3}, y_{4}, y_{7}\right)=(-$ $0.006003,4916.411329,2.686636)$ for the $4^{\text {th }}$ point, which are not the singular points of $1^{\text {st }} \mathrm{EM}$ since $y_{7} \neq 0$. Around these points, the trajectory can be approximated by ellipsis given by (eq. 18,20).

In Figures 2, the projection of the trajectory in $\left(y_{3}, y_{4}\right)$-plane superimposed with the analytical solutions around the ICs (using eq. 10,11) and around the four points that the trajectory is approaching the $3^{\text {rd }} \mathrm{EM}$ (using eq. 17, 20), are depicted.

Examining Fig. $2 b$ becomes evident that the trajectory around the ICs is very well approximated by hyperbolas (Fig. 2b) and the linearized system has real eigenvalues (eq. 5). When the trajectory is approaching points 2 and 4 the trajectory is very well approximated with ellipses (fig. $2 \mathrm{~d}$, f) which correspond to purely complex eigenvalues given by eq. (6).In cases that the trajectory is approaching points 1 and 3 the trajectory is approximated in a very small region around them (fig. $2 \mathrm{c}, \mathrm{e}$ ) with ellipses, again with zero $L E$.

The definition of Lyapunov exponent for an equilibrium of an autonomous dynamical system is given by [9],

$L E_{j}=\lim _{t \rightarrow \infty} \frac{1}{t} \operatorname{Re}\left(\sigma_{j} t\right)$,

whereas, the real part of $\sigma_{j}$ which is the $j$-eigenvalue of the linearized system around the equilibrium is considered and this limit exists when they are monotonic. In the considered case, the eigenvalues are dependent on states. When the part of the orbit is close to ICs $\left(1^{\text {st }} \mathrm{EM}\right)$ then the linearized system has pure real eigenvalues $\left(\lambda_{j}\right)$ and when it is considered part of the orbit nearby the points 1-4 ( $\left.{ }^{\text {rd }} \mathrm{EM}\right)$ then the eigenvalues $\left(\mu_{j}\right)$ are pure complex. Therefore, the real part of the $\sigma_{i}$ eigenvalues from the linearized systems approximating the original nonlinear system are varying within one orbit $\sigma_{1} \in$ $\left[0, \sqrt{\frac{I_{1} L\left(\dot{\theta}_{0}^{2}-\omega_{T}^{2}\right)}{\left(I_{1} L-F^{2}\right)}}\right]$ and the limit of eq. (21) has no specific value but since the trajectory is approximated well locally with different curves which corresponds to different eigenvalues, it can be claimed that the $L E$ is variant within an orbit. The existence of positive real eigenvalues in some parts of the orbit means nearby orbits at this region are diverging, and it is an indication of chaos.

Examining Figure 2a on the right-hand side with the indication of the eigenvalues of the $1^{\text {st }} \mathrm{EM}$ and based on the standard stability theory then the point, of $\dot{\theta}_{0}=\omega_{T}$ whereas the eigenvalues from purely complex becomes real, can be considered as the 'threshold' to discriminate the chaotic $\left(\dot{\theta}_{0}>\omega_{T}\right)$ with regular regions $\left(\dot{\theta}_{0}<\omega_{T}\right)$. Herein this is not the case. If the initial conditions are taken from the same trajectory but from the 
'lower region' $\left(\dot{\theta}_{0}<\omega_{T}\right)$ whereas the eigenvalues are purely complex, these initial conditions correspond to the regular region, but they follow a chaotic trajectory.

The analysis of the projection of the trajectory to $\left(y_{3}, y_{7}\right)$-plane superimposed with the analytical solutions provided similar results.
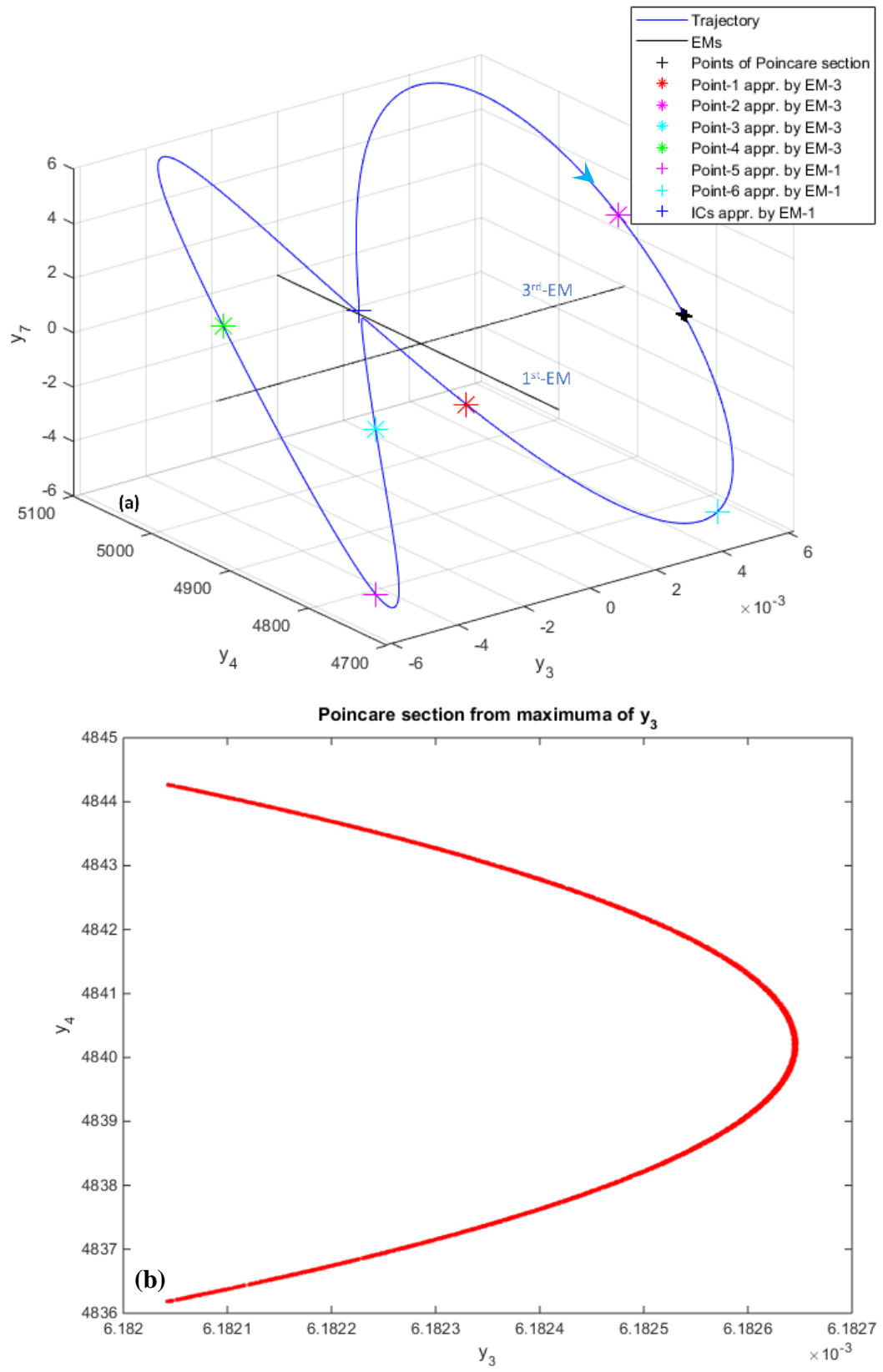
Fig. 1. (a) The total trajectory in phase space superimposed with the EMs. (b) Poincare section with maxima of torsional angle.

In Figure 1b the Poincare section obtained with zero crossings of torsional velocity $\left(y_{7}\right)$ restricted to the maxima of torsional angle $\left(y_{3}\right)$ is depicted. It is evident the absence of periodic and quasiperiodic motion due to the existence of irregular points in the Poincare section.

The numerical determination of the $L E$ has been done by three methods, in $1^{\text {st }}$ one using direct integration of the system the Lyapunov spectrum with the re-orthonormalization Gram-Schmidt (G-S) method is determined [11]. In the other two methods only, the maximum $L E$ is determined and in the $2^{\text {nd }}$ the direct integration of the system with rescaling to the neighbouring trajectory as described by Seydel in Ref. [12] is used, and in the last one, the maximum $L E$ is obtained from data series after direct numerical integration of the system using a Matlab tool [13]. The first two methods are well explained in the literature [11,12] and they are well known so it will not be explained further on. Both methods provide reliable results as long as the elementary volume expansion or contraction (an indication of $L E$ ) in the considered direction of the dynamical system, is monotonic with time [15]. Noted that in Ref. [15], in the implementation of the different numerical schemes there is an emphasis on how to implement the different schemes to avoid the non-monotonicity in time of the used estimators.

In the $3^{\text {rd }}$ method, a Matlab tool to determine the $L E$ has been used [13]. Using as input; the data series, the lag (estimated from the same tool) and the embedded dimension of the phase space then, the analysis can be performed with a tool-figure to obtain the largest $L E$. It is based in the practical method developed in [16] and the algorithm used for the determination is explained very well in [13].

Briefly, the method and the algorithm are based to curve fitting of the divergence of nearby points $\left(Y_{n}\right.$ and $\left.Y_{n^{*}}\right)$ [13],

$L D I V=\ln \frac{\left\|Y_{n+K}-Y_{n^{*}+K}\right\|}{\left\|Y_{n}-Y_{n^{*}}\right\|}$,

by considering the values of them at several time instants $(K)$ belonging to a certain expansion range $\left[K_{\min }, K_{\max }\right]$, with a plot of the average divergence then, the $L E$ estimation is done through a curve fitting in selected expansion ranges, which is based on the following formula,

$L E(n)=\frac{1}{K_{\text {max }}+K_{\min }+1} \sum_{K=K_{\min }}^{K_{\max }} \frac{1}{K \cdot d t} \ln \frac{\left\|Y_{n+K}-Y_{n^{*}+K}\right\|}{\left\|Y_{n}-Y_{n^{*}}\right\|}$.

In case that the $L E$ is variant, for different time instants $(K)$ the logarithm in eq. (22, 23) can have a positive or negative value (slope), since the ratio of the initial difference (denominator) with the consider time instants difference (nominator) throughout the expansion range is decreasing or increasing. Noted that also this method is developed for monotonic values of divergence.

All the codes for $L E$ determination have been tested successfully to obtain the benchmark values for many systems indicated in Ref. [14]. 
The determination of the $L E$ has been done using approx. 1500 orbits for the first two methods and for the last one approx. 1000 orbits. In the presenting results for the first method, it has been used $1.25 \mathrm{e}-3 \mathrm{sec}$ time step, in the $2^{\text {nd }} 4.17 \mathrm{e}-5 \mathrm{sec}$ with initial perturbation 1e-9 (limited only to torsional velocity), and in $3^{\text {rd }} 2.502 \mathrm{e}-5 \mathrm{sec}$.

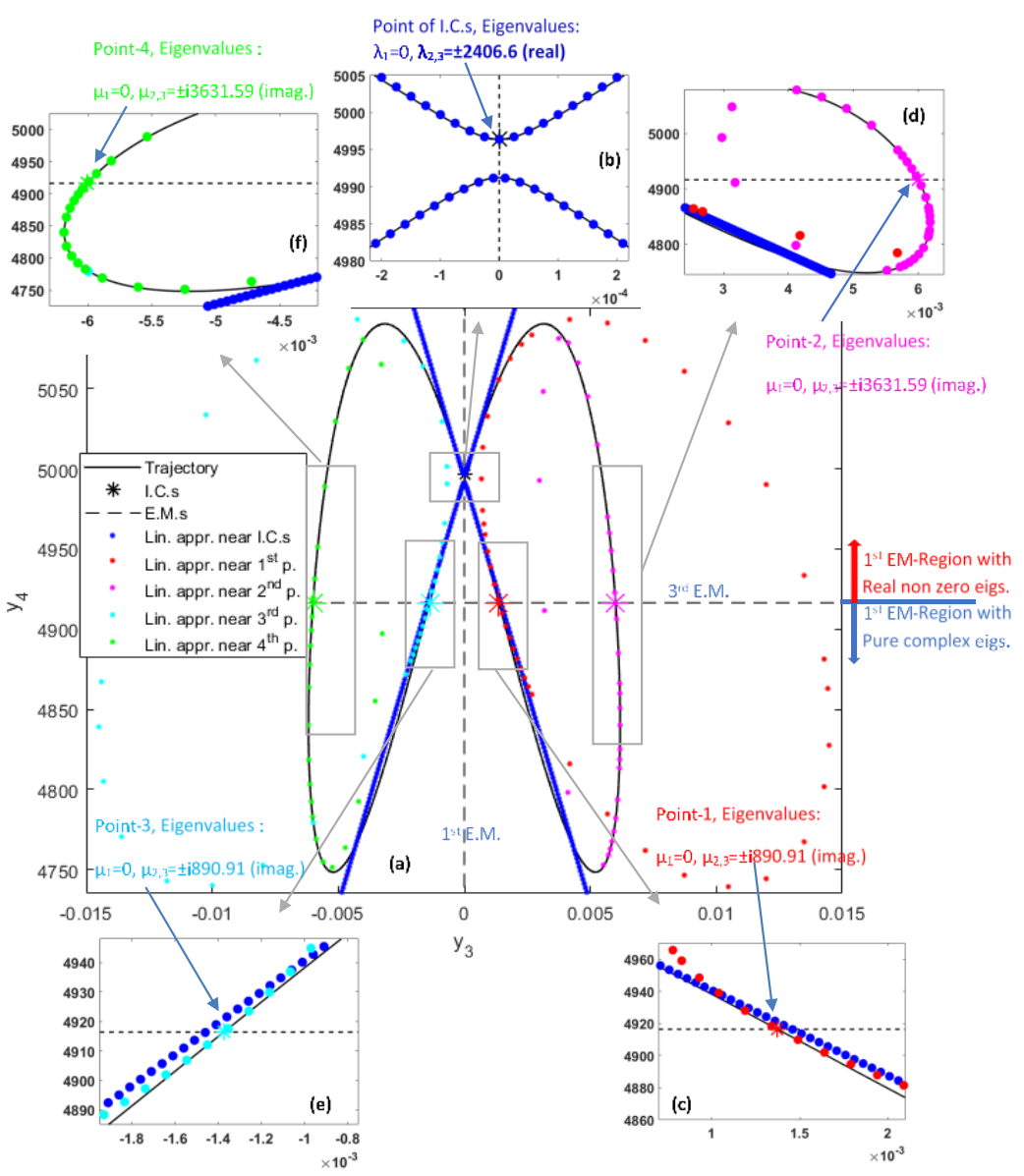

Fig. 2. Projection of the trajectory in $\left(y_{3}, y_{4}\right)$-plane superimposed with the analytical solutions around; the ICs (zoom out in Fig.2b), also of the four points that the trajectory is approaching the $3^{\text {rd }}$ EM (zoom out in Fig.2c-f).

The $L E$ spectrum obtained using the G-S method is summarized in Table- 1 whereas the largest value is positive. The accuracy of the G-S method is dependent; on the number of orthonormalizations and the choice of the finite-time step for each orthonormalization. In Hamiltonian systems, the validity of $L E$ spectrum can be checked by the sum of $L E$ which should be equal to zero, and this is the case for the spectrum in Table-1. Therefore, under the monotonic assumption of the elementary volume expansion or contraction and based on the current knowledge, the selected time step and number of 
orbits (for a specific time step is an indicator of the number of orthonormalizations) are leading to 'reliable' results.

In Table- 2 is presented a positive value of the maximum $L E$ using the $2^{\text {nd }}$ method. According to Ref. [12,14], based on reported experiments the $2^{\text {nd }}$ method $L E$ estimation, in case of monotonic divergence of nearby trajectories and if the perturbation is very small (1e-9 in this case), the maximum $L E$ is determined independently of the choice of time steps.

Table 1. Lyapunov spectrum using G-S method.

\begin{tabular}{|c|c|c|c|c|c|c|c|}
\hline In & $\mathbf{1}$ & $\mathbf{2}$ & $\mathbf{3}$ & $\mathbf{4}$ & $\mathbf{5}$ & $\mathbf{6}$ & $\mathbf{7}$ \\
\hline $\boldsymbol{L} \boldsymbol{E}_{\boldsymbol{n}}$ (bits/orbit) & 87.6 & 3.9 & 1.9 & 0 & -1.9 & -3.9 & -87.6 \\
\hline
\end{tabular}

Also, in the plots of identifying the plateaus of the $L E$ values in $1^{\text {st }}$ and $2^{\text {nd }}$ methods, there are small fluctuations within an orbit.

The Matlab tool in the $3^{\text {rd }}$ method is providing the opportunity to examine whether there are enough data points by reconstructing the phase space, which has been done successfully. In Figure 3 is depicted the plot as a Matlab tool to determine the slopes of the divergence of nearby points with respect to the expansion steps which defines the maximum $L E$ using this method. In this fig. (3) there are 4 different slopes at different expansion ranges based on the divergence of nearby points throughout the considered total expansion range (600). The estimated slopes are summarized in Table-2, noted that they have positive and negative values.

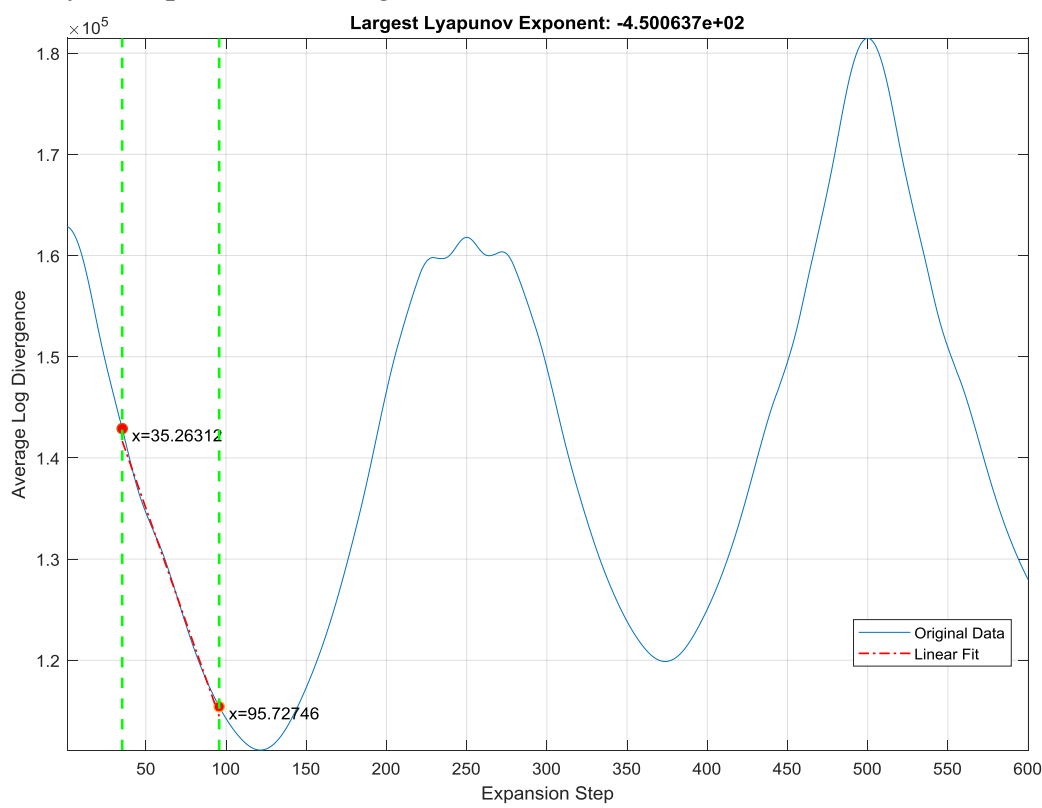

Fig. 3. Matlab tool ( $3^{\text {rd }}$ method), divergence of nearby points with respect to expansion steps. 
The values of the largest $L E$ obtained from the 3 methods are summarized in Table2 which are different. In the first two methods, the maximum $L E$ is positive but different to each other because since the $L E$ is variant within an orbit the two methods are using a different way to obtain a representative value of $L E$ throughout the total range.

In the third method, depending on the selected expansion range (time instants) then, the $L E$ have positive and negative values. The variant Lyapunov exponent arising from the theoretical analysis is also justified with this $3^{\text {rd }}$ method, whereas the different ranges correspond to different time instants that the exponent of the divergence of the orbits is varying from negative to positive.

The Matlab tool from Ref. [13] applied in the data series estimates that the approximated Information Entropy is 0.155 . Therefore, the irregularity is high, and the information loss is significant which is an indication of chaos.

Table 2. Maximum $L E$ (bits/orbit) using the three different methods.

\begin{tabular}{|l|c|c|c|c|c|c|}
\hline & G-S & $\begin{array}{l}\text { Seydel, } \\
{[\mathbf{1 2}]}\end{array}$ & $\begin{array}{l}\text { Range-1 } \\
(\mathbf{3 5 - 9 6}),[\mathbf{1 3}]\end{array}$ & $\begin{array}{l}\text { Range-2 } \\
(\mathbf{1 5 7 - 2 2 4})\end{array}$ & $\begin{array}{l}\text { Range-3 } \\
\mathbf{( 2 7 5 - 3 4 2})\end{array}$ & $\begin{array}{l}\text { Range-4 } \\
\mathbf{( 4 1 3 - 4 7 4 )}\end{array}$ \\
\hline $\boldsymbol{L E}$ & 87.6 & 13.7 & -8.1 & 11.3 & -9.8 & 10.3 \\
\hline
\end{tabular}

\section{Conclusions}

On this article chaotic dynamics of a spinning shaft with non-constant rotating speed is examined through linearization around the EM. The analytical approximation of the orbit is approximated well, only locally, nearby the different equilibriums, and, in that respect, it is shown that there are positive and zero eigenvalues which are indicative of the Lyapunov exponent and, although the limit is not existing, the Lyapunov exponent can be considered as variant within one orbit. Also, it is shown that the 'threshold' of the eigenvalues from purely complex becomes real which it should be expected to separate chaotic with regular regions in a given orbit is not valid. Therefore, a more advanced theory is needed to identify the thresholds of the chaotic motion in case of the spinning shaft. Examination of the Poincare section indicates irregular motion. The $L E$ has been determined numerically with three methods and they are very different. In the first two cases, they are positive. In third method they are clearly state-dependent with the largest being positive which certifies the variant theoretical Lyapunov exponent. Also, the approximated Information Entropy is relatively high which is an indication of chaos. Since the Lyapunov exponent is varying within an orbit the methods of calculating $L E$ that are relying on monotonicity and they are based on 'sampling' they cannot provide reliable results. This work is a first attempt to identify chaotic motions in spinning shafts using analytical and numerical methods, but further work is needed. It highlights the need for further development in the mathematics of nonlinear dynamical systems associated with EMs and the significance of the variant Lyapunov exponent examination for this kind of system. Also, generate the need to reexamine the cases of the validity of algorithms to estimate variant $L E$ within an orbit, since most of them are based on the constant value assumption, therefore there is a need of the development of new ones considering variant $L E$. 


\section{Acknowledgements}

I would like to thank and express my gratitude to Prof. Yuri V. Mikhlin for providing me valuable feedback, and to Prof. Giuseppe Rega, for his valuable comments, both on a draft manuscript.

I would like to thank and express my gratitude to the Organization Committee of $5^{\text {th }}$ Summer School of Nonlinear Dynamical Systems (1992) at University of Crete (Greece) and especially to Prof. Tassos Bountis and Prof. Spyros Pnevmatikos that they accepted my participation, although I was still undergraduate student in engineering. Since then, I followed several of them in Greece, and the knowledge that I gained, thanks to the instructors, was my induction to chaos which is substantial to write this article.

\section{References}

1. Natsiavas, S. (1995), On the dynamics of rings rotating with variable spin speed, Nonlinear Dynamics, 7: 345-363.

2. Warminski, J., Balthasar, J.M., Brasil, R.M.L.R.F. (2001), Vibrations of a non-ideal parametrically and self-excited model, Journal of Sound and Vibration, 245: 363-374.

3. Georgiades, F., Latalski, J., Warminski, J. (2014), Equations of motion of rotating composite beam with a non-constant rotation speed and an arbitrary preset angle, Meccanica, 49: 18331858. doi:10.1007/s11012-014-9926-9.

4. Georgiades, F. (2018), Nonlinear dynamics of a spinning shaft with non-constant rotating speed, Nonlinear Dynamics, 93:89-118. https://doi.org/10.1007/s11071-017-3888-0

5. Georgiades, F. (2018), Equilibrium points with their associated normal modes describing nonlinear dynamics of a spinning shaft with non-constant rotating speed, Journal of Vibration Testing and System Dynamics, 2: 327-373.

6. Liebscher, S. (2015), Bifurcation without parameters, Springer International Publishing Switzerland.

7. Guckenheimer, J., Holmes, P. (1983), Nonlinear Oscillations, Dynamical Systems, and Bifurcations of Vector Fields, Springer-Verlag New York, Inc.

8. Wiggins, S. (2003), Introduction to Applied Nonlinear Dynamical Systems and Chaos, Springer-Verlag New York, Inc.

9. Nayfeh, A.H., Balachadran, B. (2004), Applied Nonlinear Dynamics, Wiley-VCH Verlag $\mathrm{GmbH} \& \mathrm{Co} . \mathrm{KGaA}$, Weinheim.

10. Vittal, P.R. (2013), Analytical Geometry 2D and 3D, Dorling Kindersley (India) Pvt. Ltd.

11. Benettin G., Galgani, L, Giorgilli, A., Strelcyn, J.-M. (1980), Lyapunov Characteristic Exponents for Smooth Dynamical Systems and for Hamiltonian System; A Method for Computing all of them, Meccanica 15: 21-30.

12. Seydel, R. (2010), Practical Bifurcation and Stability Analysis, Springer Science+Bussiness Media, LLC NY.

13. Mathworks, Matlab R2018b, (2018), Predictive Maintenance Toolbox, website http://www.mathworks.com/products/

14. Wolf, A., Swift, J.B., Swinney, H.L., Vastano, J.A. (1985), Determining Lyapunov exponents from a time series, Physica D 16:285-387.

15. Geist, K., Parlitz, U., Lauternborn, W. (1990), Comparison of Different Methods for Computing Lyapunov Exponents, Progress of Theoretical Physics 83:875-893. 
16. Michael T. Rosenstein, James J. Collins, Carlo J. De Luca, (1993), "A practical method for calculating largest Lyapunov exponents from small data sets ". Physica D 65:117-134. 